\title{
High-Pressure Chemistry of a Zeolitic Imidazolate Framework Compound in the Presence of Different Fluids
}

\author{
Junhyuck $\mathrm{Im}^{1}$, Narae Yim $^{2}$, Jaheon $\mathrm{Kim}^{2}$, Thomas Vogt, ${ }^{3}$ and Yongjae Lee ${ }^{1,4, *}$ \\ ${ }^{1}$ Department of Earth System Sciences, Yonsei University, Seoul 03722, Korea. \\ ${ }^{2}$ Department of Chemistry, Soongsil University, Seoul 06978, Korea. \\ ${ }^{3}$ NanoCenter and Department of Chemistry \& Biochemistry, University of South Carolina, \\ Columbia, South Carolina 29208, United States \\ ${ }^{4}$ Center for High Pressure Science \& Technology Advanced Research (HPSTAR), Shanghai \\ 201203, China.
}

\section{Corresponding Author}

*Email: yongjaelee@yonsei.ac.kr, Tel.:+82-2-2123-5667. 


\section{PART I. Experimental Details}

Synthesis of ZIF-8. Zinc nitrate tetrahydrate $\left(\mathrm{Zn}\left(\mathrm{NO}_{3}\right)_{2} \cdot 6 \mathrm{H}_{2} \mathrm{O} ; 0.146 \mathrm{~g}, 0.49 \mathrm{mmol}\right)$ was dissolved in $10 \mathrm{~mL}$ methanol. Separately, 2-methylimidazole $(0.325 \mathrm{~g}, 3.96 \mathrm{mmol})$ was also dissolved in $10 \mathrm{~mL}$ methanol. The zinc salt solution was poured quickly into the ligand solution, and the resulting mixture was slowly stirred for 2 hours at room temperature. The white precipitates were collected by centrifuging the product mixture. The collected solid was washed with neat methanol $(3 \times 25 \mathrm{~mL})$ and dried at $50{ }^{\circ} \mathrm{C}$ in a convection oven for 24 hours. ${ }^{1}$

Synchrotron X-ray diffraction. In situ synchrotron X-ray powder diffraction experiments were performed at the beamline 10-2 at the Stanford Synchrotron Radiation Lightsource (SSRL) and the beamline 5A at the Pohang Accelerator Laboratory (PAL). At the SSRL, a focused X-ray beam with a wavelength of 0.6199(1) A was used. The XRD patterns of ZIF-8 in the presence of different PTM (Table s1) were obtained using a Pilatus 300K-w direct photon counting detector. The detector exhibits gaps near 8.2 and 12.8 degrees as indicated in Figure $2 \mathrm{a}, 2 \mathrm{~b}$ and $2 \mathrm{~d}$. At beamline $5 \mathrm{~A}$ at the PAL, monochromatic hard X-ray photons emitted from an in-vacuum undulator source were monochromatized to a wavelength of 0.6926(1) $\AA$ and were detected using a MAR-345 imaging plate detector. The wavelength of the incident beam and the detector calibrations were carried out using $\mathrm{LaB}_{6}$ standard reference material (SRM 660b) at both synchrotron facilities.

High-pressure experiments. A modified Merrill-Bassett type diamond anvil cell (DAC) with two opposed diamonds supported by tungsten-carbide plates ${ }^{2}$ was used for the highpressure XRD measurements. The anvils used were brilliant-cut type-1A diamonds with a culet diameter of $700 \mu \mathrm{m}$. The DAC has a rectangular asymmetric slot on one side to provide an opening of ca. 40 degrees, through which diffraction data are measured. The powdered sample was loaded into a $350 \mu \mathrm{m}$ diameter and less than $150 \mu \mathrm{m}$ thick sample chamber obtained by electro-spark erosion in a pre-indented stainless steel foil gasket. A few ruby spheres of $\sim 10 \mu \mathrm{m}$ diameter were added as a pressure gauge. Subsequently, a powdered sample of as-synthesized ZIF-8 was added to the sample chamber and either water, methanol, 
ethanol or silicone oil was added as a hydrostatic pressure transmitting media (PTM). The pressure at the sample in the DAC was measured by detecting the shift of R1 emission line of included ruby spheres (precision: $\pm 0.05 \mathrm{GPa}$ ). ${ }^{3}$ The pressure was calculated using the equation below (1):

$$
P=A / B\left[1+\left(\Delta \lambda / \lambda_{0}\right)\right]^{B}
$$

where $P$ is the pressure in megabars, $\lambda$ is the wavelength of the ruby $R$ line, $A=19.04$ Mbar, $B=7.665 .{ }^{4}$ The sample was equilibrated for about 10 minutes in the DAC at each measured pressure. Changes in the unit cell lengths and volume were derived from a series of whole profile fitting procedure using the GSAS suite of programs. ${ }^{5-6}$ 


\section{Part II. Supporting Figure}

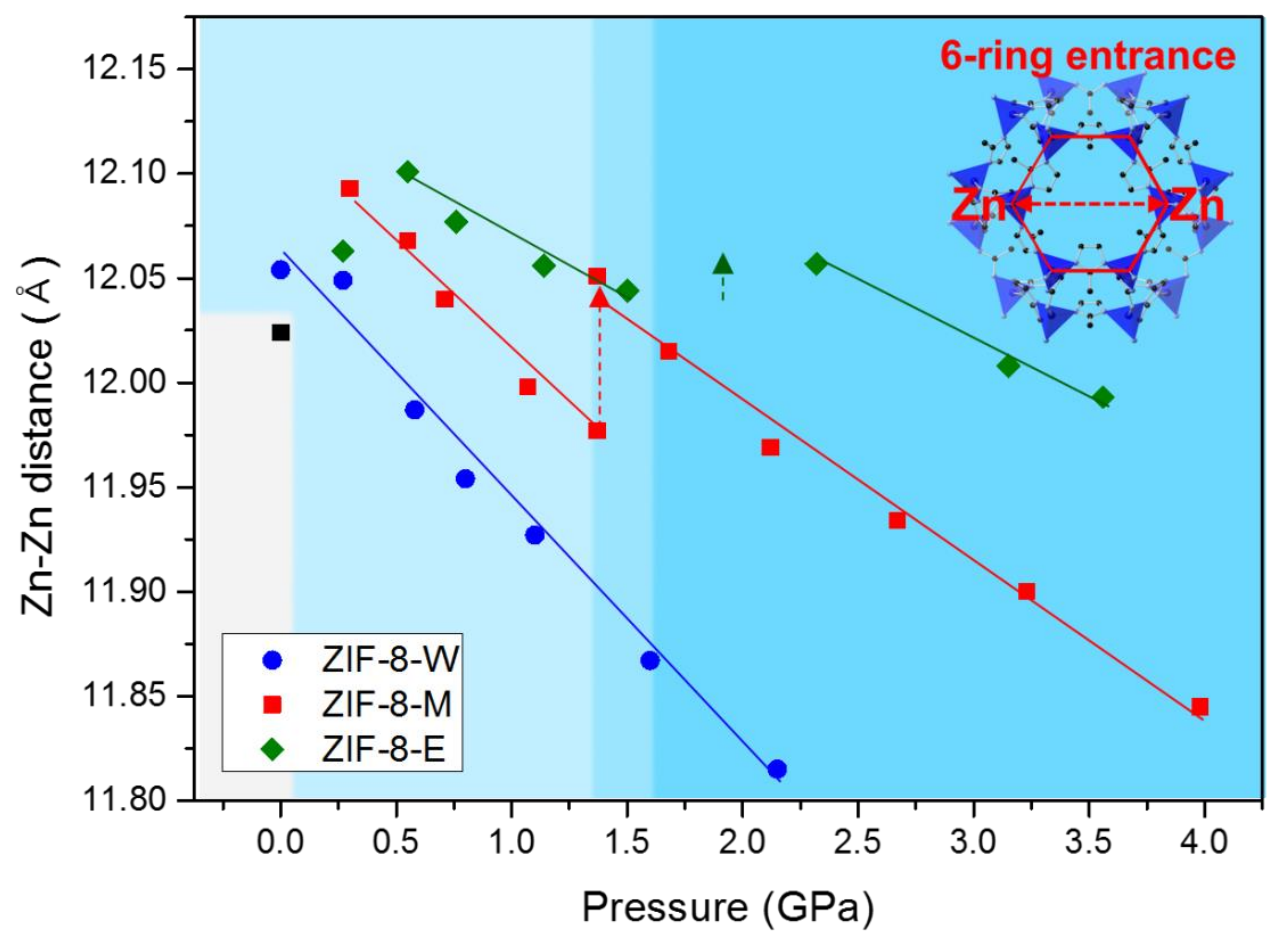

Figure S1. Pressure-dependent changes of Zn-Zn distance in the 6-ring entrance of the ZIF8 framework in the presence of water (W), methanol (M), and ethanol (E) pressure transmitting medium conditions. 


\section{Part III. Supporting Tables}

Table S1. Summary of the pressure-dependent behavior of ZIF-8 with various pressure transmitting media (PTM).

\begin{tabular}{ccccc}
\hline \hline PTM & $\mathrm{P}_{\mathrm{t}}(\mathrm{GPa})^{\mathrm{a}}$ & $\beta\left(\mathrm{GPa}^{-1}\right)^{\mathrm{b}}$ & $\mathrm{K}_{0}(\mathrm{GPa})^{\mathrm{c}}$ & Synchrotron Facilities $^{\text {water }}$ \\
methanol & 1.4 & 0.03143 & 41.71 & $\mathrm{BL10-2}$ at SSRL \\
ethanol & 2.3 & 0.02703 & $40(2)$ & $\mathrm{BL} 10-2$ at SSRL \\
silicone oil & & 0.01754 & $73(4)$ & $\mathrm{BL}$ A at PAL \\
\hline
\end{tabular}

${ }^{a} \mathrm{P}_{\mathrm{t}}$ : pressure of the phase transition

${ }^{\mathrm{b}} \beta$ : compressibility of ZIF-8-PII $(1-x)$ phases

${ }^{c} \mathrm{~K}_{0}$ : bulk moduli (BM) of ZIF-8 (silicone oil PTM) and ZIF-8-PII phases ( $\mathrm{K}_{0}$ ': fixed at 4) 
Table S2. Changes of the unit cell parameters, volumes, residual electron density and inserted guest molecules as a function of pressure in ZIF-8. ${ }^{\mathrm{a}}$

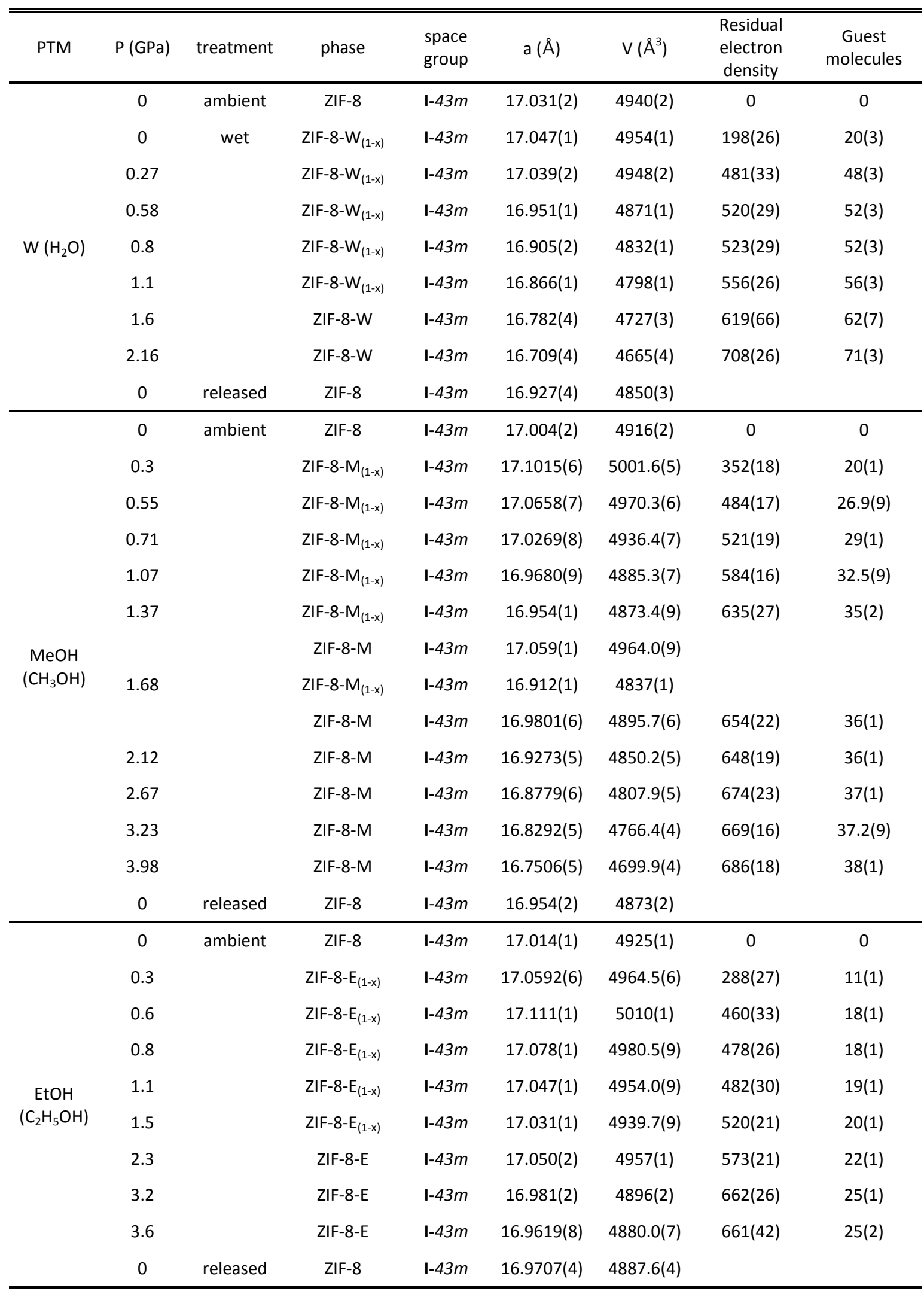




$\begin{array}{ccccccc} & 0 & \text { ambient } & \text { ZIF-8 } & \text { I-43m } & 17.031(2) & 4940(2) \\ \text { Silicone } & 0.3 & & \text { ZIF-8-O } & \text { I-43m } & 17.013(1) & 4925(1) \\ \text { Oil } & 0.5 & & \text { ZIF-8-O } & \text { I-43m } & 16.892(4) & 4820(4)\end{array}$

${ }^{\text {a } E S D ' s ~ a r e ~ i n ~ p a r e n t h e s e s . ~}$ 


\section{Part IV. References}

(1) Cravillon, J.; Münzer, S.; Lohmeier, S.-J.; Feldhoff, A.; Huber, K.; Wiebcke, M. Chem. Mater. 2009, 21, 1400.

(2) Mao, H. O. K.; Hemley, R. J. Philos. Trans. A Math. Phys. Eng. Sci. 1996, 354, 1315.

(3) Bell, P. M.; Mao, H. K. Absolute pressure measurements and their comparison with the ruby fluorescence $(R 1)$ pressure scale to 1.5 Mbar; Carnegie Institution: Carnegie Institution of Washington Year Book, 1979; pp 665-669.

(4) Mao, H. K.; Xu, J.; Bell, P. M. J. Geophys. Res.-Solid 1986, 91, 4673.

(5) Larson, A. C.; Dreele, R. B. V. GSAS-General Structure Analysis System; Los Alamos National Laboratory: 1986.

(6) Toby, B. H. J. Appl. Crystallogr. 2001, 34, 210. 